\title{
Dependence of Modulation Bandwidth on Size of Optical Injection Locked Semiconductor Ring Laser
}

\author{
M.I. MEmon and H. FATHallah \\ Prince Sultan Advanced Technologies Research Institute (PSATRI), King Saud University, Riyadh, Saudi Arabia
}

\begin{abstract}
It has been successfully investigated for the first time that strength of optical injection locking also depends on the size of semiconductor ring laser. Theoretical study for frequency response of optical injection locking semiconductor ring laser in the master slave configuration using direct, amplitude and phase modulation of master laser is discussed. In the unidirectional regime the locking range of semiconductor ring laser becomes wider when semiconductor ring laser with smaller size is used. Simulation results predict an incredible enhancement in the modulation bandwidth $(>500 \mathrm{GHz})$ of the slave semiconductor ring laser when used with smaller laser cavity length $(50 \mu \mathrm{m})$ in the stable unidirectional regime.
\end{abstract}

DOI: $10.12693 /$ APhysPolA.125.462

PACS: 42.55.Wd, 42.65.Re, 42.60.By, 42.65.Pc

\section{Introduction}

To fulfill the requirements of future high speed communication system a lot of research effort has been invested to develop high speed laser diodes and optical modulators over last two decades. For application as the light source, in communications, modulation bandwidth is a key issue. This is more valid for emerging complex modulation based communication that involves both amplitude and phase in (quadrature amplitude modulation) with coherent detection. The highest direct modulation bandwidth of free-running laser reported so far is about $30 \mathrm{GHz}[1]$.

Optical injection locking (OIL) in semiconductor lasers (SLs) [2] has been found suitable in achieving single-mode operation, frequency chirp reduction, noise reduction and in achieving large enhancement in the frequency response at $3 \mathrm{~dB}$ bandwidth [3]. Previously frequency response of the master-slave configuration of OIL lasers has been discussed both experimentally and theoretically. It has been shown that the $3 \mathrm{~dB}$ bandwidth of OIL slave lasers can be considerably increased [4] in the master-slave configuration. Nevertheless, these systems are not appropriate for coplanar integration as the backward light from the slave has to be stopped from entering the master laser using an isolator [5].

Semiconductor ring laser (SRL) is a promising slave laser device for integrated OIL master-slave configuration while modulating the master laser, as the SRL works only in the direction receiving injection, therefore does not feedback into the master laser. In Refs. [5, 6], we have already demonstrated enhancement in the bandwidth.

Very fast switching time in terms of picoseconds makes the SRL a potential candidate for all optical signal processing $[7,8]$. Besides different dimensions and designs, simple and integrated SRLs are reported. Directional bistability is a key characteristic of SRL [9] and a variety of applications are based on it including all-optical logic [9], label swapping [10], all-optical memory [11], and optical regeneration [12]. The phenomena of OIL and four-wave mixing [13] have been reported in the SRL at particular conditions. A variety of applications has been reported using these two phenomena such as bandwidth enhancement [5,6], all-optical multicast [14], all-optical logic gates [15] and millimetre wave ( $\mathrm{mm}$ wave) generation and modulation [16].

In this letter for the first time to the best our knowledge, enhancement in the bandwidth of SRL due to decrease in its size is proposed. Hence the smaller is the size the stronger will be OIL. Stable locking region becomes larger with the decrease in the cavity length of slave OIL-SRL. Theoretical analysis of rate equation of OIL-SRL using direct, amplitude and phase modulation of the master laser is discussed in detail. Simulation analysis using different sizes of SRL predicts modulation bandwidth of $>500 \mathrm{GHz}$. It can be very vital for $400 \mathrm{Gbps}$ optical transmission systems.

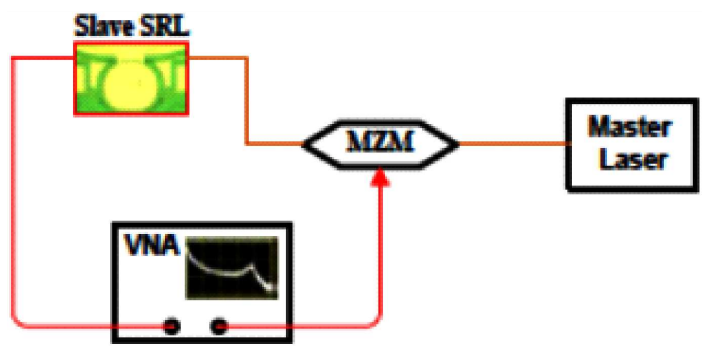

Fig. 1. Illustration of master slave bandwidth enhancement system using SRL.

The master slave bandwidth enhancement system using SRL as slave laser is illustrated in Fig. 1. The signal from the network analyser is modulated with the (continuous wave) light coming from the master laser (ML) using a modulator. SRL is made locked with this modulated signal. Output from the SRL is passed back to the network analyser to measure the frequency response. 


\section{Theory}

When the bistable SRL works in the unidirectional region, its direction can be controlled by the injection of light from the master laser. The single mode rate equations as defined in [6] can be used to describe the injection locked SRL. Using the steady state solution and small signal analysis [6], the frequency response of slave SRL due to direct current modulation of the master laser is the rate of change of output photon density of slave SRL to the change in the bias current of the master laser and it can be given as

$$
H_{\mathrm{dir}}(\mathrm{j} \omega)=\frac{\delta S}{\delta I}=M_{\mathrm{dir}} \frac{\mathrm{j} \omega+Z_{d}}{\left(\mathrm{j} \omega+\omega_{\mathrm{p}}\right)\left(\omega_{R}^{2}-\omega^{2}+\mathrm{j} \omega \gamma\right)} .
$$

Similarly, frequency response of the SRL due to intensity modulation of the master laser can be given as

$$
H_{\mathrm{AM}}(\mathrm{j} \omega)=\frac{\delta S}{\delta S_{\mathrm{inj}}}=M_{\mathrm{AM}} \frac{\left(\mathrm{j} \omega+Z_{\mathrm{AM} 1}\right)\left(\mathrm{j} \omega+Z_{\mathrm{AM} 2}\right)}{\left(\mathrm{j} \omega+\omega_{\mathrm{p}}\right)\left(\omega_{R}^{2}-\omega^{2}+\mathrm{j} \omega \gamma\right)} .
$$

Equations (1), (2) have the same denominator which can be separated into a first order and a second order response, thus characterized by the same resonance frequency $\omega_{R}$ and damping factor $\gamma$. In free-running lasers these are decided by laser material and structural parameters, as well as steady state parameters such as pump current density and photon density, and have been extensively investigated previously. However, in OIL systems, they are also highly dependent on the injection strength $R_{\text {inj }}$ defined as the ratio between the injected photon density and the cavity photon density, and the detuning $\Delta \omega_{\text {inj }}$ between the injection optical frequency and the laser mode free-running frequency.

The decrease in the cavity size of the SRL affects $\tau_{\mathrm{p}}$ (photon life-time), $\tau_{1}$ (cavity round trip time) and $\mathrm{V}$ (volume of the active region). These quantities can be defined as

$$
\begin{aligned}
& \tau_{\mathrm{p}}=\frac{\Gamma v_{\mathrm{g}}}{L_{\mathrm{cav}}} \log \left(\frac{1}{1-T}\right) \Rightarrow \tau_{\mathrm{p}} \propto \frac{1}{L_{\mathrm{cav}}}, \\
& \tau_{1}=\frac{L_{\mathrm{cav}}}{v_{\mathrm{g}}} \Rightarrow \tau_{1} \propto L_{\mathrm{cav}}, \\
& V=L_{\mathrm{cav}} W_{\mathrm{wg}} T_{q w} N_{q w} \Rightarrow V \propto L_{\mathrm{cav}},
\end{aligned}
$$

where $L_{\text {cav }}$ is cavity size of SRL, if the size is decreased, $\tau_{\mathrm{p}}$ is increased as a result the output photon density is also increased. Similarly $\tau_{1}$ is directly proportional to the $L_{\text {cav }}$, however when $\tau_{1}$ is reduced $\kappa_{\text {inj }}$ is enhanced. As a result the detuning frequency becomes stronger. All these factors play key role when the SRL of smaller size is used as a slave laser in the master slave configuration and its modulation bandwidth becomes stronger as compared to the larger cavity size SRL.

\section{Simulation}

Simulation is based on the SRL device very similar to used in $[5,6]$, however different cavity sizes of SRL are assumed. Figure 2 illustrates stable locking range for different cavity lengths of SRL.

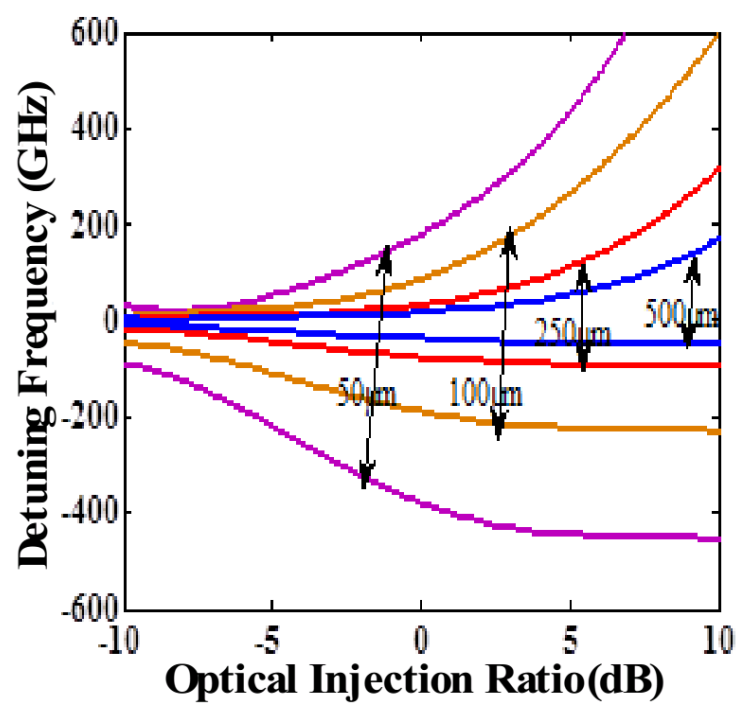

Fig. 2. Stable locking range of SRL with different cavity sizes.

As the focus of this paper is the comparison of injection locking between different sizes of SRL, thus only stable locking range is shown in Fig. 2. It demonstrates very clearly that stable locking regions widens with the decrease in the size of SRL. It is not only affecting the volume of the active region, it is also proportional to the photon lifetime and gain of the laser. $50 \mu \mathrm{m}$ is used as the smallest size of SRL because stable unidirectional region is assumed [17, 18].

Equations (1)-(3) are simulated using four distinct cavity lengths i.e. 50, 100, 250, and $500 \mu \mathrm{m}$, respectively. Figure 2 shows the frequency response of SRL to the external direct current modulated light. The drawback in direct modulation of OIL lasers is the roll-off factor from the extra pole as given in Eq. (1) that affects the bandwidth. Using amplitude and phase modulation this effect can be eliminated.

OIL can also be affected by the size of the SRL. Equations (1), (2) are simulated with four different cavity lengths i.e. 50, 100, 250, and $500 \mu \mathrm{m}$, respectively.

Figure 3 shows the frequency response of OIL SRL using direct modulation, the drawback in direct modulation of OIL lasers is the roll-off factor from the extra pole as given in Eq. (1) that affects the bandwidth.

Using amplitude modulation of the master laser this effect can be eliminated. Figure 4 demonstrates the frequency response of OIL SRL using amplitude modulation of the master laser. In all of these responses the optical injection ratio is kept constant to $10 \mathrm{~dB}$. Due to reduction in size the detuning frequency becomes stronger. Thus modulation bandwidth is enhanced and predicted $>500 \mathrm{GHz}$. 


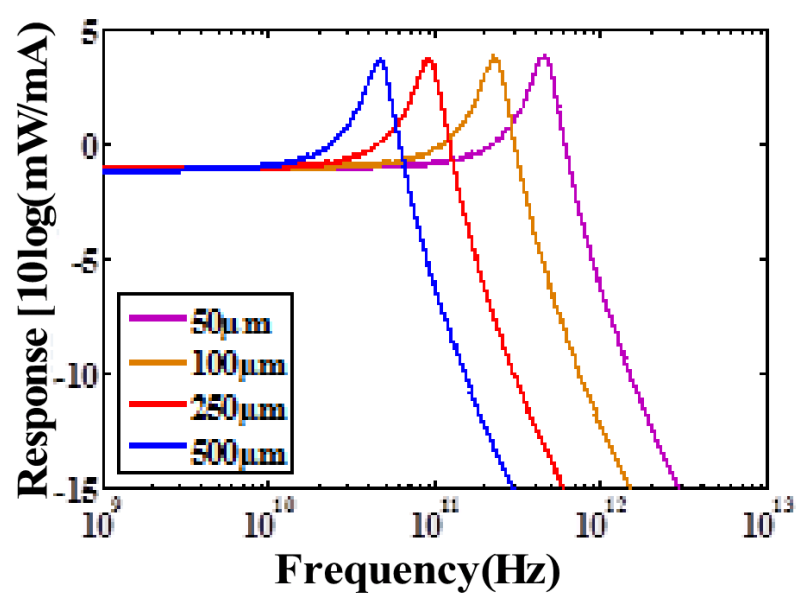

Fig. 3. Frequency response of SRL to external direct modulated light using different cavity sizes.

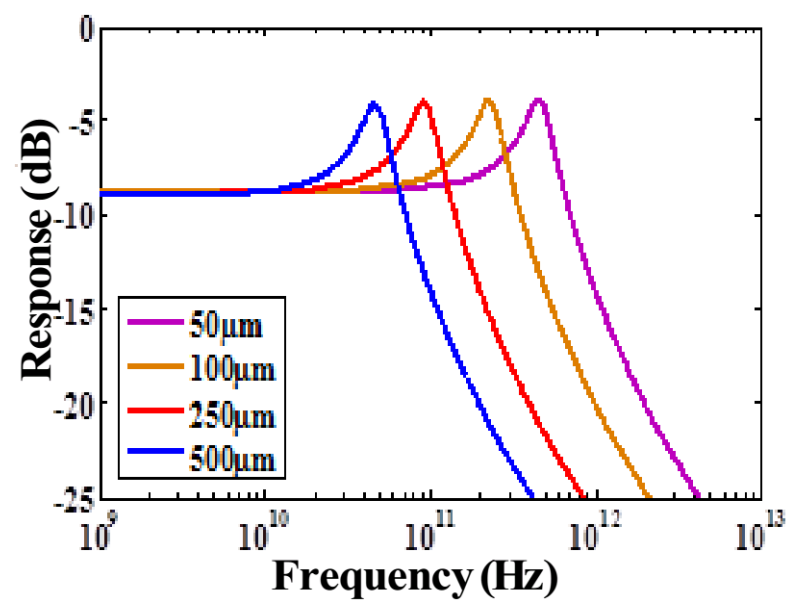

Fig. 4. Frequency response of SRL to external amplitude modulated light using different cavity sizes.

\section{Conclusion}

In this paper we have investigated the dependence of OIL in SRLs on its cavity length. Theoretical model for frequency response of OIL slave SRL using direct, amplitude and phase modulation of master laser is discussed in detail. It is simulated using different cavity lengths and proved successfully that OIL also depends on the laser size. If the length of SRL cavity is reduced to $50 \mu \mathrm{m}$, incredible enhancement in the modulation bandwidth of
$>500 \mathrm{GHz}$ is predicted. It makes SRL a key element in future high speed communication system. Additionally, the proposed scheme readily offers itself for monolithic integration as already exhibited in [18]. This type of integrated device may provide a useful component for high bandwidth data communications applications.

\section{References}

[1] Y. Matsui, H. Murai, S. Arahira, Y. Ogawa, A. Suzuki, IEEE J. Quant. Electron. 34, 1970 (1998).

[2] R. Lang, IEEE J. Quantum Electron. 18, 976 (1982).

[3] E.J. Bochove, J. Opt. Soc. Am. B 14, 2381 (1997).

[4] E.K. Lau, L.J. Wong, X.X. Zhao, Y.-K. Chen, C.J. Chang-Hasnain, M.C. Wu, IEEE J. Light Wave Technol. 26, 2584 (2008).

[5] M.I. Memon, B. Li, G. Mezosi, Z. Wang, M. Sorel, S. Yu, IEEE Photon. Technnol. Lett. 21, 1792 (2009).

[6] M.I. Memon, H. Fathallah, S. Yu, Acta Phys. Pol. A 123, 180 (2013).

[7] M. Sorel, G. Giuliani, A. Scire, R. Miglierina, S. Donati, P.J.R. Laybourn, IEEE J. Quantum Electron. 39, 1187 (2003).

[8] C. Born, M. Sorel, S. Yu, IEEE J. Quantum Electron. 41, 261 (2005).

[9] B. Li, M.I. Memon, G. Mezosi, Z. Wang, M. Sorel, S. Yu, J. Opt. Commun. 30, 190 (2009).

[10] K. Thakulsukanant, B. Li, S. Furst, M. Sorel, S. Yu, CLEO, San Jose, CThH7 (2008).

[11] M.T. Hill, H.J.S. Dorren, T.J. de Vries, X.J.M. Leijtens, J.H. den Besten, E. Smalbrugge, Y.S. Oei, G.D. Khoe, M.K. Smit, Nature 432, 206 (2004).

[12] B. Li, M.I. Memon, G. Mezosi, Z. Wang, M. Sorel, S. Yu, IEEE J. Lightwave Technol. 27, 4233 (2009).

[13] S. Fürst, M. Sorel, IEEE Photon. Technol. Lett. 20, 366 (2008).

[14] D. Lu, G. Mezosi, B. Li, M.I. Memon, Z. Wang, M. Sorel, S.S. Jian, S. Yu, IET Electron. Lett. 44, 1374 (2008).

[15] B. Li, D. Lu, M.I. Memon, G. Mezosi, Z. Wang, M. Sorel, S. Yu, IET Electron. Lett. 45, 698 (2009).

[16] M.I. Memon, B. Li, G. Mezosi, Z. Wang, M. Sorel, S. Yu, IEEE Photon. Technol. Lett. 21, 733 (2009).

[17] G. Mezösi, M.J. Strain, S. Furst, Z. Wang, S. Yu, M. Sorel, IEEE Photon. Technol. Lett. 21, 88 (2009).

[18] S. Fürst, S. Yu, M. Sorel, IEEE Photon. Technol. Lett. 20, 1926 (2008). 